\title{
Isolamento social, uso de álcool e as notificações de violência física em uma Região do
} Nordeste Brasileiro, 2016-2021

\author{
Social isolation, alcohol use and reports of physical violence in a region of Northeast Brazil, 2016-
} 2021

Aislamiento social, uso de alcohol y relatos de violencia física en una región del Nordeste de Brasil, 2016-2021

\section{Resumo}

O isolamento social e o uso de álcool relacionam-se com as notificações e, portanto demandam uma vigilância em saúde contínua visto que, eleva o risco da ocorrência dos casos de violência física em mulheres. Objetivou-se então, analisar o isolamento social e as notificações de violência física em uma Região Nordeste Brasileiro. Estudo ecológico descritivo a respeito das notificações de violência física em mulheres no Ceará. A coleta de dados ocorreu na base de dados do SINAN/DATASUS, no período de 2016 a 2021. Foram notificados 8.754 casos de violência física destacando-se o perfil das vítimas na raça parda 6.593, escolaridade: ensino médio incompleto: 1944. Houve predominância de consumo de bebida alcoólica no agressor da violência em 3.714 casos. As notificações apontaram um decréscimo significativo no ano de 2021 implicando com o período de isolamento social na pandemia de COVID19.

Palavras-chave: Notificação Compulsória; Sistema de Informações de Agravos de Notificação (SINAN); Violência contra a mulher.

\footnotetext{
Abstract

Social isolation and alcohol use are cases of notification and therefore require continuous health surveillance since the risk of physical violence in women. To analyze social isolation and reports of physical violence in a Brazilian Northeast Region. Descriptive ecological study about reports of physical violence against women in Ceará. The collection of teaching data in the SINAN/DATASUS database, from 2016 to 2021 and 8,754 cases of physical violence in education were reported, with the profile of victims in the brown race 6,593, schooling: incomplete high school: 1944 .There was a predominance of alcohol consumption in the aggressor of violence in 3,714 cases. The
} 
notifications pointed to a significant decrease in the year 2021, implying the period of social isolation in the COVID19 pandemic.

Keywords: Compulsory Notification; Notifiable Diseases Information System (SINAN); Violence against women.

\section{Resumen}

El aislamiento social y el consumo de alcohol son casos de notificación y por lo tanto requieren vigilancia continua de la salud dado el riesgo de violencia física en las mujeres. Analizar el aislamiento social y las denuncias de violencia física en una región del Nordeste brasileño. Métodos: Estudio ecológico descriptivo sobre denuncias de violencia física contra la mujer en Ceará. La recolección de datos docentes en la base de datos SINAN/DATASUS, de 2016 a 2021. Se reportaron 8.754 casos de violencia física en la educación, con perfil de víctimas en la raza parda 6.593, escolaridad: bachillerato incompleto: 1944. Hubo predominio del consumo de alcohol en el agresor de violencia en 3.714 casos.Las notificaciones apuntaron a una disminución significativa en el año 2021, lo que implica el período de aislamiento social en la pandemia de COVID-19.

Palabras clave: Notificación Obligatoria; Sistema de Información de Enfermedades de Declaración Obligatoria (SINAN); Violencia contra las mujeres.

\section{Introdução}

As estimativas da Organização Pan-Americana de Saúde demonstraram a magnitude da COVID-19 mediante a propagação do vírus SARS-COV-2, em meados do ano de 2020, onde foram evidenciados 85.380 casos confirmados e 5.901 óbitos no Brasil (Opas, 2020).

A dualidade entre o isolamento social imposta em decorrência como medida de mitigação do avanço dos casos de COVID-19 por Lei n ${ }^{\circ} 13.979$ e a permanência de um período de convivência interrupto entre o agressor e a mulher vítima de violência física culminou no aumento dos casos (Brasil, 2020; Lettiere-Viana, 2021).

Assim como no Brasil, a tendência do aumento dos casos de violência física contra a mulher a partir do fator condicionante do isolamento social durante a pandemia COVID-19 foi registrada na Espanha, Itália, França e China (Siqueira e Miranda, 2021).

Logo, o objetivo deste estudo é analisar a relação entre o isolamento social o uso e álcool e as notificações de violência física em mulheres na Região do Nordeste: Ceará.

\section{Metodologia}

Estudo ecológico, descritivo com abordagem quantitativa das notificações de violência física em mulheres no Ceará. A coleta de dados ocorreu na base de dados do Sistema de Informação de Agravos de Notificação ( SINAN), em sua versão disponibilizada off-line do Ministério da Saúde aos municípios brasileiros, versão esta conhecida por SINAN-net por meio do site DATASUS (Brasil,2022). O corte temporal mediante as notificações ocorreu no período de 2016 a 2021.

O método de pesquisa do tipo quantitativo possibilita a observação e análise apurada dos dados estaticamente a partir de medidas mensuráveis numéricas ((Pereira, et.al.; Estrela, 2018).

A população da pesquisa consistiu no registro das fichas de notificações de violência física das mulheres a partir de 15 anos até maiores de 60 anos de idade residentes nos 184 municípios do estado do Ceará (Ceará, 2022).

Variáveis da Tabulação on-line através do Tabnet: Uf de notificação: Ceará, Faixa etária: de 15 a maiores de 60 anos de idade, Sexo: Feminino, Raça: Todas as Categorias e Violência Física: Sim (Brasil, 2022).

Os critérios de exclusão: Lesão autoprovocada, Enforcamento, Envenenamento, e Própria Pessoa (Brasil, 2022).

\section{Resultados e Discussão}

A evolução das notificações da violência física em mulheres na série história de 2016 a 2021 correspondeu a totalidade de 8.754 ocorrências. Em 2019, momento até então pré-pandêmico de COVID-19 houve significância de 2.468 notificações e uma redução acentuada em 2020 com 1.843 e no ano de 2021 apresentou 946 registros (Tabela 1). 
Tabela 1: Frequência da violência física em mulheres de 15 a maiores de 60 anos por ano de notificação segundo Região Nordeste/Uf de notificação: Ceará,2022.

\begin{tabular}{|l|l|l|l|l|l|l|l|}
\hline Região Nordeste/ Uf de Notificação: Ceará & 2016 & 2017 & 2018 & 2019 & 2020 & $* 2021$ & Total \\
\hline Ceará & 740 & 922 & 1.835 & 2.468 & 1.843 & 946 & 8.754 \\
& & & & & & & \\
\hline
\end{tabular}

Fonte: Ministério da Saúde/SVS - Sistema de Informação de Agravos de Notificação - Sinan Net.

O perfil sociodemográfico das vítimas destacou-se a raça parda 6.593, seguida da branca 1.261. Ressalta-se ainda a falha durante o preenchimento das notificações diante da demonstração de 220 fichas ignoradas ou em branco, dificultando a definição da raça determinante para a construção do perfil sociodemográfico (tabela 2).

Tabela 2: Frequência por Raça segundo violência física em mulheres de 15 a maiores de 60 anos por ano de notificação segundo Região Nordeste/Uf de notificação: Ceará, 2022.

\begin{tabular}{|l|l|l|l|l|l|l|l|}
\hline Região Nordeste & Ignorado/Branco & Branca & Preta & Amarela & Parda & Indígena & Total \\
\hline Ceará & 220 & 1.261 & 599 & 48 & 6.593 & 33 & 8.754 \\
\hline
\end{tabular}

Fonte: Ministério da Saúde/SVS - Sistema de Informação de Agravos de Notificação - Sinan Net.

Destacou-se assim, a raça parda apresentou 6.593, branca 1.261 e preta 599 notificações (tabela2). A raça é o fenótipo da pele humano também conhecido na população brasileira por tipo de cor de pele: branca, preta, amarela, parda e indígena. Ao qual, provocada uma discussão entre as classes sociais devido a povos miscigenados onde cada indivíduo se autodeclara por motivos pessoais, culturais e alguns discordam da classificação oficial emitida na declaração de óbito cabendo ações de ordem jurídica para reverter o devido fato ( Santos e Maio,2004 ; Pinho e Sansone, 2008; Orth e Graf,2020).

Tabela 3: Frequência por escolaridade segundo violência física em mulheres de 15 a maiores de 60 anos por ano de notificação segundo Região Nordeste/Uf de notificação: Ceará,2022.

\begin{tabular}{|l|l|l|l|l|l|l|l|l|l|l|l|l|}
\hline Região & Ignorado/Branco & Analfabeto & $\begin{array}{l}1^{\mathrm{a}} \mathrm{a} 4^{\mathrm{a}} \\
\text { incompleto } \\
\mathrm{EF}\end{array}$ & $\begin{array}{l}1^{\mathrm{a}} \mathrm{a} 4^{\mathrm{a}} \\
\text { completo } \\
\mathrm{EF}\end{array}$ & $\begin{array}{l}5^{\mathrm{a}} \mathrm{a} 8^{\mathrm{a}} \\
\text { incompleto } \\
\mathrm{EF}\end{array}$ & $\begin{array}{l}\text { Ensino } \\
\text { fundamental } \\
\text { completo }\end{array}$ & $\begin{array}{l}\text { Ensino } \\
\text { médio } \\
\text { completo }\end{array}$ & $\begin{array}{l}\text { Ensino } \\
\text { médio } \\
\text { incompleto }\end{array}$ & $\begin{array}{l}\text { Superior } \\
\text { Completo }\end{array}$ & $\begin{array}{l}\text { Superior } \\
\text { incompleto }\end{array}$ & $\begin{array}{l}\text { Não } \\
\text { se } \\
\text { Aplica }\end{array}$ & $\begin{array}{l}\text { Total } \\
\text { Ceará }\end{array} 1.992$ \\
\hline
\end{tabular}

Fonte: Ministério da Saúde/SVS - Sistema de Informação de Agravos de Notificação - Sinan Net. Atualizados em *16/10/2022

A apresentação dos dados corroboram o ensino médio incompleto com 1.944 registros na escolaridade das mulheres vítimas de violência, em sequência do período correspondente da $5^{\mathrm{a}}$ a $8^{\mathrm{a}}$ série do ensino fundamental incompleta com $1.270 \mathrm{e}$ $1^{\mathrm{a}}$ a $4^{\mathrm{a}}$ do ensino fundamental incompleto por meio de 695 casos(tabela 3 ).

O grau de instrução contribui como um medidor dos casos de violência física ao relacionar os fatores socioeconômicos da mulher na qual a maioria dos casos surge a tendência em que quanto mais anos de estudos maiores serão as oportunidades de inserção no mercado de trabalho e consequentemente sua independência financeira aumentando assim as chances encerrarem os vínculos com os agressores por não serem dependentes dos mesmos economicamente (Caetano et.al., Rodrigues et.al, 2021). 
Cabe à sociedade ofertar uma educação básica e superior de qualidade gratuita, além de facilidades de inserção no mercado de trabalho para que meninas antes de se tornarem mulheres conscientizem-se dos seus valores e não se sujeitem a situações de violências (Leite e Silva, 2021).

O amparo das vítimas deve ocorrer nos casos de emergência em casos de abrigos nos alojamentos conjuntos para mulheres e seus filhos, exemplificando, a Casa da Mulher Brasileira na qual são fornecidos serviços multidisciplinares de assessoria jurídica, serviço social, enfermagem, nutrição entre outros. Além de proteção, auxílio financeiro e a inserção da vítima em oficinas de trabalhos autônomos visando sua autossuficiência (Preparo, 2021).

Tabela 4: Frequência por Suspeito uso de álcool segundo violência física em mulheres de 15 a maiores de 60 anos por ano de notificação segundo Região Nordeste/Uf de notificação: Ceará, 2022.

\begin{tabular}{|l|l|l|l|l|l|}
\hline Região Nordeste & Sim & Não & Ignorado & Em Branco & Total \\
\hline Ceará & 3.714 & 3.320 & 1.673 & 47 & 8.754 \\
\hline
\end{tabular}

Fonte: Ministério da Saúde/SVS - Sistema de Informação de Agravos de Notificação - Sinan Net. Atualizados em *16/10/2022.

Os achados implicam que a maioria os agressores que cometeram violência física contra as mulheres somam-se a 3.714 casos utilizaram da ingestão do álcool por meio de bebidas alcóolicas antes de cometerem a agressão(tabela 4).

O isolamento social contribui para o agressor estar acompanhado da vítima em um período contínuo e prolongado de horas diárias impostas por uma medidas de mitigação do SARS-COV-2 bem como a ingestão do consumo de álcool com o intuito de diminuir os casos de coronavírus, em contrapartirda impõe a vítima a potencialização dos mais diversos tipos de violência, inclusive a violência física(Oliveira et.al; Santos, 2021).

Dessa forma, a exposição da violência além de traumas psicológicos, danos físicos resultantes das agressões que inclusive possam acarretar em alterar a locomoção motora dessa mulher em casos de tetraplegia necessitando de gastos e suporte médico diário e por todo o ciclo de vida, e em casos culminem no óbito(Soares, et.al; Barbosa et.al, 2021).

O monitoramento das notificações de violência devem ser permanentes nas três esferas de gestão e ofertar as vítimas os mais diversos instrumentos de proteção assegurados por leis específicas no enfrentamento dos casos de violência(Vieira et al; Correa, 2021).

\section{Conclusão}

As notificações de violência física foram sujeitas a fatores predisponentes para o aumento dos casos como o isolamento social e uso de álcool, porém a estatística apresentada no ano de 2021 contendo 946 casos demonstra justamente a contradição em representar um índice abaixo do esperado visto que, surge a preocupação em analisar além dos números onde pode estar havendo subnotificações ou mesmo esse decréscimo inclusive pode ser devido ao fato do agressor em coibir a mulher vítima de violência física em denunciar.

Outro fato, está relacionado à diminuição das ofertas e serviços de saúde apresentando o direcionamento de atendimento aos pacientes acometidos por COVID-19 comprometendo assim a prestação da assistência médica as vítimas de violência física. 
Logo, sugere-se que além da disposição dos dados recomenda-se ao pesquisador um olhar holístico da dinâmica do contexto relacionado aos fatores condicionantes para a elevação dos dados e contrapor com o quantitativo para assim, elaborar hipóteses que afirmem ou contestem os resultados baseados em evidências científicas.

\section{Referências}

Organização Pan-Americana da Saúde (OPAS) / OMS (2020). Folha informativa: COVID-19 (doença causada pelo novo coronavírus).Atualizada em 03 de maio de 2020. https://www.paho.org/bra/index.php?option=com_content\&view=article\&id=6101:covid19\&Itemid=875.

Brasil. Lei no 13.979, de 6 de fevereiro de (2020). http://www.in.gov.br/en/web/dou/-/lei-n-13.979-de-6-de-fevereiro-de-2020-242078735.

Barbosa, J. P. M., Lima, R. D. C. D., Santos, G. D. B. M., Lanna, S. D., \& Andrade, M. A. C. (2021). Interseccionalidade e violência contra as mulheres em tempos de pandemia de covid-19: diálogos e possibilidades. Saúde e Sociedade, 30.

Brasil.Ministério da Saúde. (2022). Datasus. Violência interpessoal e autoprovocada. http://www.tabnet.datasus.gov.br/cgi/tabcgi.exe?sinannet/cnv/violebr.def Brasil.Ministério da Saúde. (2022). Tabnet. Violência interpessoal e autoprovocada. http://www.tabnet.datasus.gov.br/cgi/tabcgi.exe?sinannet/cnv/violebr.def

Caetano da Silva, A. J. ., Belchior de Medeiros, E. ., Silva Basílio, I. C. ., Alves Barbosa, J. K. ., \& Egidio da Silva, R. . (2021). Vítimas de maus-tratos, negligência ou abandono em estado do Nordeste Brasileiro. Nursing (São Paulo), 24(273), 5289-5298. https://doi.org/10.36489/nursing.2021v24i273p52895298 .

Correa, J. D. S. (2021). As projeções do avanço em saúde mental nos casos de tabagismo, álcool e violência doméstica no cenário pandêmico da covid-19: uma revisão sistemática da literatura (Doctoral dissertation).

Teixeira S.M.LA e Miranda CFGHR (2021). Mulheres em isolamento social: a problemática do aumento de casos de violência doméstica em tempos de pandemia. Revista Crimialis.ed 1, p.12,v1..

Estrela, C. (2018). Metodologia Científica: Ciência, Ensino, Pesquisa. Editora Artes Médica.

Pereira.A.S. . S. et al. (2018). Metodologia da pesquisa científica.UFSM.

Pinho, AO., and Sansone, L., orgs.( 2008).Raça: novas perspectivas antropológicas [online]. (2nd ed. rev.) EDUFBA, 447 p. ISBN 978-85-232-1225-4. Available from SciELO Books

Rodrigues PA, Cicolella DA, Mariot MDM (2021) Prevalência de violência contra a mulher e suas repercussões na maternidade. J. nurs. health.;11(1): e2111119459. https://periodicos.ufpel.edu.br/ojs2/index.php/enfermagem/article/view/19459

Santos, R.V e Maio, M.C. (2004).Qual "retrato do Brasil"? Raça, biologia, identidades e política na era da genômica. Mana [online]. v. 10, n. 1 [Acessado 16 Janeiro 2022] , pp. 61-95. https://doi.org/10.1590/S0104-93132004000100003. Epub 30 Jun 2004. ISSN 1678-4944. https://doi.org/10.1590/S010493132004000100003

Ceará. Secretaria da Saúde(2022). https://www.saude.ce.gov.br/.

Lettiere-Viana, A. et al. Coping strategies for violece against children, adolescentes and women in the contexto of social isolation due to COVID-19: scoping review.(2021) Texto \& Contexto - Enfermagem [online]. , v. 30 [Acessado 15 Janeiro 2022] , e20200443. https://doi.org/10.1590/1980-265X-TCE-20200443>. Epub 26 Abr 2021. ISSN 1980-265X. https://doi.org/10.1590/1980-265X-TCE-2020-0443.

Orth, N.M.G e Graf, M.P.Sulear a justiça restaurativa: as contribuições latino-americanas para a construção do movimento restaurativo/ [livro eletrônico]/ (Org.). Ponta Grossa: Texto e Contexto, 2020. (Coleção Singularis, v.8) 292 p.; e-book PDF Interativo.

Leite M e Silva, C.A.(2021).Um estudo sobre o papel da mulher nasociedade brasileira por meio de O Cravo e a Rosa e Coisa Mais Linda. v. 34 n. 55 (2021): 50 anos da Transamazônica

Oliveira, M. M., Magalhães, F. E. M., Oliveira, A. L. R., \& Júnior, M. A. B. (2021). Consequências do isolamento social na pandemia. Bionorte, 10(S2).

Peraro, B. C. (2021). Rede de atendimento à mulher e um olhar sobre o programa Casa da Mulher Brasileira.

Santos, N. A. D. D. (2021). Violência doméstica nos tempos de isolamento social.

Soares, C. F., da Silva Araújo, R., Estrela, F. M., Morais, A. C., Farias, R. V., de Almeida, V. R. S., ... \& da Cruz, N. R. S. (2021). Fatores precipitantes e/ou agravantes da violência contra crianças no contexto da COVID-19/Precipitating and/or aggravating factors of violence against children in the context of COVID-19. Brazilian Journal of Development, 7(1), 9430-9442.

Vieira, M. B. W., Feitosa, F. E. A., de Freitas Pinheiro, D., da Silva, L. G., \& de Alcantara, P. P. T. (2021). Novas formas de denunciar casos de violencia domestica durante a quarentena propiciada pelo covid-19. Holos, 3, 1-11. 